\title{
Treatment patterns and long-term persistence with osteoporosis therapies in women with Medicare fee-for-service (FFS) coverage
}

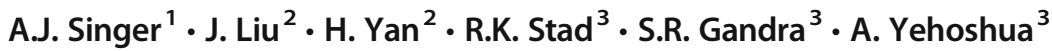 \\ Received: 18 December 2020 / Accepted: 5 April 2021 / Published online: 7 June 2021 \\ (C) The Author(s) 2021
}

\begin{abstract}
Summary Osteoporosis, a chronic disease, requires long-term therapy. In Medicare-insured women, denosumab persistence was higher than oral bisphosphonate persistence over up to 3 years of follow-up. Longer-term persistence was higher among women who persisted in the first year of therapy.

Introduction Osteoporosis, a chronic, progressive disease, requires long-term therapy; this study assessed long-term persistence with anti-resorptive therapies in postmenopausal women.

Methods This retrospective cohort study used administrative claims for women with data in the $100 \%$ Medicare osteoporosis sample who initiated (index date) denosumab, oral/intravenous (IV) bisphosphonate, or raloxifene between 2011 and 2014 and who had $\geq 1$ year (zoledronic acid: 14 months) of pre-initiation medical/pharmacy coverage (baseline). Persistence was assessed from index date through end of continuous coverage, post-index evidence of censoring events (e.g., incident cancer), death, or end of study (December 31, 2015).

Results The study included 318,419 oral bisphosphonate users (78\% alendronate), 145,056 denosumab users, 48,066 IV bisphosphonate users, and 31,400 raloxifene users; mean age ranged from 75.5 years (raloxifene) to 78.5 years (denosumab). In women with at least 36 months of follow-up (denosumab $N=25,107$; oral bisphosphonates $N=79,710$ ), more denosumab than oral bisphosphonate initiators were persistent at 1 year (73\% vs. 39\%), 2 years (50\% vs. 25\%), and 3 years (38\% vs. 17\%). Persistence decreased over time for all treatment groups, with denosumab users having the highest persistence in every follow-up time interval at or after 18 months. Women using denosumab, oral bisphosphonates, or raloxifene who persisted in a given year were more likely to remain persistent through the subsequent year.

Conclusions Denosumab users persisted longer with therapy than women using other anti-resorptive medications, including oral bisphosphonates. Early persistence may predict long-term persistence. Overall persistence with osteoporosis medications is suboptimal and may impact fracture risk. Efforts to improve first year persistence are needed.
\end{abstract}

Keywords Denosumab · Osteoporosis · Persistence $\cdot$ Postmenopausal women $\cdot$ Treatment patterns

\section{Introduction}

Osteoporosis is a chronic and progressive disease which occurs disproportionately in postmenopausal women, with this

A. Yehoshua

alony@amgen.com

1 MedStar Georgetown University Hospital and Georgetown University Medical Center, Washington, DC, USA

2 Chronic Disease Research Group (CDRG), Hennepin Healthcare Research Institute, Minneapolis, MN, USA

3 Global Health Economics, Amgen, Inc., Thousand Oaks, CA, USA at-risk population growing rapidly as the US population ages and life expectancy increases [1,2]. Osteoporosis is largely asymptomatic until a fragility fracture occurs, at which point the consequences can be devastating and may include ongoing pain, loss of functionality and independence, impaired quality of life, or even death [3-8]. Individuals and society overall incur significant direct and indirect costs from these fragility fractures $[5,9,10]$.

Osteoporosis is generally underdiagnosed and undertreated despite the wide availability of effective pharmacologic treatments designed to reduce the risk of fracture $[6,11]$. In the USA, oral bisphosphonates are the most commonly prescribed initial treatments for postmenopausal women with osteoporosis [12], although intravenous (IV) bisphosphonates, raloxifene, 
denosumab, teriparatide, abaloparatide, and romosozumab are also approved for this indication. Better persistence (i.e., continuing to use a medication for the prescribed duration [13]) is generally associated with greater treatment benefit (fracture risk reduction). Two early real-world studies reported a 26-29\% greater fracture risk reduction in persistent alendronate patients compared to their non-persistent counterparts over 24 months of follow-up $[14,15]$. This association between persistence and greater fracture reduction has more recently been documented across a broad range of medications used in treating postmenopausal osteoporosis [16].

Despite the increased risk of fracture due to non-persistence, more than half of patients with postmenopausal osteoporosis discontinued therapy within the first year of initiating an oral bisphosphonate [17]. Non-persistent patients remain at elevated risk of fracture and related consequences $[8,18,19]$. Medication characteristics including dosing frequency and complexity of dosing instructions, along with fear of side effects, and the degree to which patients accurately understand fracture risk and the benefits/risks of treatment, shape patients' ability to persist with osteoporosis therapies [20]. While less-frequent dosing and electronic prescriptions have been shown to improve persistence, patient education and monitoring efforts have not $[14,20,21]$. A systematic review published in 2020 found that only a limited number of studies have reported that patient education, monitoring and supervision, change in drug regimen combined with patient support, and interdisciplinary collaboration have a positive effect on either adherence or persistence with osteoporosis medications [22]. The more recent literature included in this review suggests that patient involvement and shared decision-making have become more common over time and multicomponent interventions with active patient involvement may have a more positive effect, although this would need to be confirmed with future research. Some physicians de-prioritize osteoporosis treatment relative to other medical conditions [23-25] which, in turn, may contribute to undertreatment and to patients' beliefs that osteoporosis is of lesser importance.

Previous studies have demonstrated better persistence with denosumab at 12 and 24 months in the USA, Canada, and Europe [26-28] in comparison with reported persistence for oral bisphosphonates [17]. In a recent single-center study in the USA, denosumab demonstrated persistence of $51 \%$ over 36 months of follow-up and $64 \%$ of patients had 7 total injections with 1 administered every 6 months \pm 8 weeks during follow-up [29]. Given that osteoporosis is a chronic, progressive disease that requires ongoing therapy, the current study was designed to expand upon these earlier findings by assessing long-term persistence for anti-resorptive therapies in a large population of postmenopausal women in the USA with Medicare coverage.

\section{Methods}

\section{Cohort selection}

This retrospective cohort study relied on administrative claims and enrollment data from 2010 through 2015 captured for the $100 \%$ Medicare osteoporosis sample. Female Medicare beneficiaries who initiated an osteoporosis therapy of interest (denosumab, oral/IV bisphosphonate, raloxifene) between 2011 and 2014 were 66 years or older at initiation (index date) and who had at least 1 year of Medicare parts A (hospitalization, skilled nursing facility, home health services), B (physician services, outpatient care, injectable medications, durable medical equipment), and D (patient-administered pharmaceuticals) coverage (14 months required for zoledronic acid users) prior to initiation were included in the study. Patients who were treated with glucocorticoids or who had evidence of Paget's disease of the bone, osteogenesis imperfecta, hypercalcemia, or cancer during the year prior to the index date were excluded. Patients who were enrolled in a Medicare Advantage plan which was managed through a Medicare-approved private insurance company were also excluded as their medical claims may have been incompletely captured. Patients were followed from the index date through the end of their continuous Medicare coverage period, occurrence of a censoring event (e.g., postindex events that parallel study exclusion criteria, such as incident cancer diagnosis or diagnosis of Paget's disease), death, or end of study (December 31, 2015). The 12 months before the index date was used as baseline assessment period.

\section{Persistence}

Persistence was defined as the duration of continuous use of a medication from initiation to end of follow-up as defined above. Treatment non-persistence was indicated by an untreated period of 60 days or longer (discontinuation) or evidence of a switch to a different class of osteoporosis medication (denosumab, oral bisphosphonate, IV bisphosphonate, raloxifene, teriparatide, calcitonin). For oral medications, nonpersistence is defined by the absence of a refill within 60 days after the expected refill date which is based on fill date and the days' supply value for each pharmacy claim. For parenteral medications, non-persistence is defined by the absence of drug administration within 60 days after the date of the next expected injection based on the labeled dosing schedule. This gap interval was chosen as it is commonly reported in published studies of persistence with osteoporosis medications. In sensitivity analysis, this gap threshold was replaced with a value representing half of the "supply interval" which for injectable medications is defined by the labeled dosing schedule interval 
which translates to 46 days for IV ibandronate, 3 months for denosumab, and 6 months for zoledronate.

\section{Statistical analysis}

Data on baseline patient characteristics were analyzed descriptively using frequencies and percentages to summarize categorical data and means and standard deviations (SD) to summarize continuous data. Patient demographic characteristics (age, race, sex, ethnicity, and Medicare-Medicaid dual eligibility status) were determined as of the index date. Clinical characteristics including the Deyo adaptation of the Charlson Comorbidity Index (CCI) and individual comorbidities of interest (cerebrovascular accidents/transient ischemic attack, congestive heart failure [CHF], other cardiovascular disease, chronic obstructive pulmonary disease [COPD], anemia, depression), number of other medications (excluding osteoporosis medications) used, as well as fragility fracture and fall history, were quantified during the 12 month baseline assessment period.

Since the study included patients with differing lengths of follow-up, the percentage of patients who remained persistent with the index medication was determined at 6-month followup intervals through 36 months of follow-up, with results reported separately for different index treatments (denosumab, oral bisphosphonates, IV bisphosphonates, raloxifene). Subsequent year persistence was assessed for the subgroups of patients who were (1) persistent in year 1 and (2) persistent in years 1 and 2. Predictors of non-persistence were assessed using a Cox's proportional hazards regression model to adjust for patient demographics, comorbidities, baseline medication use, and prior fragility fracture. Treatment patterns (continued index medication, switched to a different class of osteoporosis medication, discontinued osteoporosis medication) over time were examined descriptively and results are represented with a flowchart.

\section{Results}

The final study population included 318,419 oral bisphosphonate users, 145,056 denosumab users, 48,066 IV bisphosphonate users, and 31,400 raloxifene users (Table 1), and the characteristics of these patients are provided in Supplementary Table S1. Approximately $78 \%$ of women in the oral bisphosphonate group used alendronate. The mean age (SD) of patients in the treatment groups ranged from 75.5 (6.9) years in women who used raloxifene at index date to 78.5 (7.4) years in denosumab users. White women comprised the majority in each treatment group (81-92\%). The mean CCI ranged from 0.49 in the raloxifene group to 0.71 in the denosumab group. At the index date, denosumab users were more likely to have a history of fragility fracture in the previous year than women in the other treatment groups (denosumab, 8.7\%; IV bisphosphonate, 7.7\%; oral bisphosphonate, $7.1 \%$; raloxifene, $4.3 \%$ ).

Although persistence with the index medication varied by medication class, within each assessment interval, persistence levels by medication type were similar for patients who had at least 3 years of follow-up and patients who were censored or lost to follow-up prior to the 3-year mark (Table 2). Among patients whose follow-up spanned the entire 3-year assessment period (denosumab $N=25,107$; oral bisphosphonates $N=79,710$; IV bisphosphonates $N=17,454$; raloxifene $N=8703$ ), persistence with the index medication decreased over time for all treatment types, with denosumab users having the highest persistence in every time interval at or after 18 months (Fig. 1). Approximately $38 \%$ of the denosumab users persisted for at least 3 years. The corresponding percentages for the other treatments were $17 \%$ for oral bisphosphonates, $6 \%$ for IV bisphosphonates, and 23\% for raloxifene. During the first 6 and 12 months on treatment, persistence in denosumab users was higher than persistence among patients using other medication classes except for IV bisphosphonates. This pattern reflects the fact that the majority (94.4\%) of patients in the IV bisphosphonate group used zoledronic acid, for which a single injection provides 12 months of persistence. Despite this high level of first year persistence, only approximately $40 \%$ of IV bisphosphonate users had received their second scheduled infusion by the 18-month assessment. Among denosumab, oral bisphosphonate, and raloxifene patients who were persistent in the first year or the first 2 years of therapy, $64 \%$ to $76 \%$ were persistent in the next year (Table 3 ). In the IV bisphosphonate group, the percentage of previously persistent patients who remained persistent in the third year of follow-up was less than half that observed in the second year of follow-up (16\% versus $36 \%$ ).

Patients who were non-persistent with the index treatment were followed to determine how many restarted treatment (i.e., re-initiated the index treatment or switched to another type of osteoporosis treatment), and how many remained untreated from that point through the end of follow-up. These results are shown in Fig. 2a-d. Approximately 40\% $(57,988)$ of all 145,056 denosumab users were non-persistent in the follow-up period. Of these non-persistent patients, $25 \%$ reinitiated denosumab after a mean gap of 167 days (i.e., 107 days after the 60-day gap that defined discontinuation) and $24 \%$ switched to a different class of osteoporosis medication (mean time to switch: 33 days after the 60-day gap that defined discontinuation). The remaining $52 \%$ of nonpersistent patients remained off osteoporosis therapy through the end of follow-up. More than half of switchers changed to their new treatment within 60 days after discontinuing their index treatment. Approximately $67 \%(n=212,684)$ of oral 
Table 1 Patient selection

\begin{tabular}{lllll}
\hline & \multicolumn{2}{l}{ Number of patients } & \\
\cline { 2 - 5 } Criterion & Denosumab & $\begin{array}{l}\text { Oral } \\
\text { bisphosphonates }\end{array}$ & $\begin{array}{l}\text { IV } \\
\text { bisphosphonate }\end{array}$ & Raloxifene \\
\hline Treatment initiation 2011-14 (index date) & 532,041 & $1,998,726$ & 234,024 & 229,395 \\
Female & 442,770 & $1,755,952$ & 209,407 & 228,429 \\
Age $>66$ and <110 years at index date & 388,103 & $1,339,411$ & 173,285 & 166,614 \\
Continuous benefits coverage for 1 year & 218,185 & 444,490 & 78,135 & 42,253 \\
$\quad$ prior to index date & 167,550 & 393,739 & 65,277 & 36,662 \\
$\quad$ No history of cancer, Paget's disease of & & & & \\
$\quad$ bone, osteogenesis imperfecta, or & & & 53,121 & 33,878 \\
$\quad$ hypercalcemia & 145,685 & 347,445 & 48,066 & 31,400 \\
No glucocorticoid use & 145,056 & 318,419 & 48,066 & 31,400 \\
Final cohort & 145,056 & 318,419 & & \\
\hline
\end{tabular}

Table 2 Percent of patients remaining persistent by length of available follow-up (full study population)

Assessment Interval

\begin{tabular}{|c|c|c|c|c|c|c|c|}
\hline & Number of Parents & 6-Month & 12-Month & 18-Month & 24-Month & 30-Month & 36-Month \\
\hline \multicolumn{8}{|l|}{ 6+ Months Follow-up } \\
\hline Denosumab & 123,833 & 93.9 & & & & & \\
\hline Raloxifene & 27,095 & 57.2 & & & & & \\
\hline IV Bisphosphonates & 40,712 & 94.7 & & & & & \\
\hline Oral Bisphosphonates & 272,470 & 56.3 & & & & & \\
\hline \multicolumn{8}{|l|}{ 12+ Months Follow-up } \\
\hline Denosumab & 107,233 & 93.9 & 73.3 & & & & \\
\hline Raloxifene & 23,882 & 57.6 & 43.0 & & & & \\
\hline IV Bisphosphonates & 35,201 & 94.8 & 93.2 & & & & \\
\hline Oral Bisphosphonates & 237,537 & 56.6 & 39.4 & & & & \\
\hline \multicolumn{8}{|l|}{ 18+ Months Follow-up } \\
\hline Denosumab & 78,026 & 93.8 & 72.9 & 60.4 & & & \\
\hline Raloxifene & 19,052 & 57.9 & 43.5 & 35.3 & & & \\
\hline IV Bisphosphonates & 30,444 & 94.9 & 93.4 & 33.7 & & & \\
\hline Oral Bisphosphonates & 184,983 & 56.7 & 39.4 & 30.2 & & & \\
\hline \multicolumn{8}{|l|}{ 24+ Months Follow-up } \\
\hline Denosumab & 55,116 & 93.6 & 72.5 & 59.7 & 50.7 & & \\
\hline Raloxifene & 14,861 & 57.7 & 43.5 & 35.5 & 30.8 & & \\
\hline IV Bisphosphonates & 26,424 & 95.1 & 93.7 & 33.9 & 33.3 & & \\
\hline Oral Bisphosphonates & 141,223 & 56.5 & 39.3 & 30.3 & 25.0 & & \\
\hline \multicolumn{8}{|l|}{ 30+ Months Follow-up } \\
\hline Denosumab & 37,381 & 93.6 & 72.6 & 59.5 & 50.3 & 43.1 & \\
\hline Raloxifene & 11,619 & 57.9 & 43.8 & 35.8 & 31.1 & 26.8 & \\
\hline IV Bisphosphonates & 23,015 & 95.4 & 94.1 & 34.3 & 33.7 & 9.0 & \\
\hline Oral Bisphosphonates & 108,307 & 56.3 & 39.0 & 30.1 & 24.9 & 20.4 & \\
\hline \multicolumn{8}{|l|}{ 36+ Months Follow-up } \\
\hline Denosumab $(n=25,107)$ & 25,107 & 93.6 & 72.7 & 59.6 & 50.3 & 43.2 & 38.0 \\
\hline Raloxifene $(\mathrm{n}=8,703)$ & 8703 & 57.5 & 43.5 & 35.5 & 30.9 & 26.5 & 23.2 \\
\hline IV Bisphosphonates $(\mathrm{n}=17,454)$ & 17,454 & 95.4 & 94.1 & 40.1 & 39.5 & 10.3 & 6.4 \\
\hline Oral Bisphosphonates $(\mathrm{n}=79,710)$ & 79,710 & 56.1 & 38.8 & 29.8 & 24.7 & 20.3 & 17.2 \\
\hline
\end{tabular}




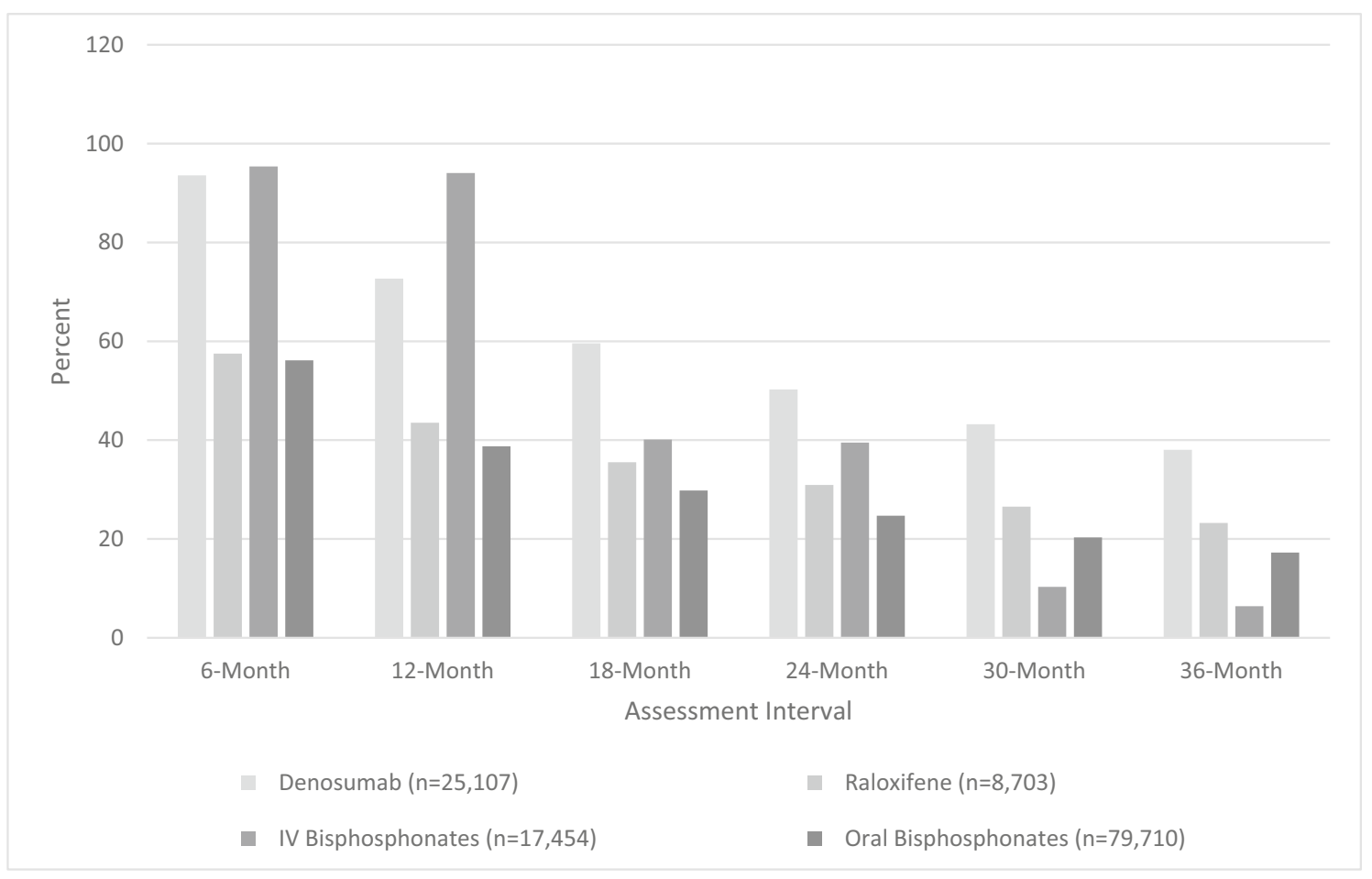

*Patients with 36 months follow-up

Fig. 1 Percent of patients remaining persistent*

bisphosphonate users did not persist with their index medication, although $35 \%$ re-initiated oral bisphosphonates prior to the end of follow-up. Nearly all others (62\% of discontinuers) had no further osteoporosis medication use, and a minority $(<4 \%$ of discontinuers) switched to a different medication. On average, 151 days elapsed between the discontinuation date and treatment re-initiation, and 127 days elapsed between the discontinuation and the switch. Among the 31,067 patients who were non-persistent with IV bisphosphonates (65\% of all 48,066 IV bisphosphonate users), $84 \%$ remained untreated through the

Table 3 Next year persistence in previously persistent patients

\begin{tabular}{lcc}
\hline Treatment & Number of patients & Percent of total \\
\hline \multicolumn{2}{l}{ Year 2 persistence in patients who persisted in year 1} \\
Denosumab & 27,914 & 69.9 \\
Raloxifene & 4574 & 70.7 \\
IV bisphosphonates & 8805 & 35.6 \\
Oral bisphosphonates & 35,304 & 63.7 \\
Year 3 persistence in patients who persisted in years 1 and 2 \\
Denosumab & 9547 & 75.7 \\
Raloxifene & 2022 & 75.1 \\
IV bisphosphonates & 1115 & 16.2 \\
Oral bisphosphonates & 13,742 & 69.8
\end{tabular}

\footnotetext{
${ }^{1}$ Denominator is the number of patients who were persistent with each specific treatment during the previous year
}

end of follow-up, 14\% switched treatments (mean time from discontinuation to switch: 191 days), and less than $2 \%$ reinitiated IV bisphosphonate (mean time from discontinuation to re-initiation: 79 days). The majority (64\%) of raloxifene users were non-persistent with their index medication with $48 \%$ of these 20,126 non-persistent raloxifene users failing to resume treatment during the remaining follow-up time, $23 \%$ switching to a new treatment (mean time from discontinuation to switch: 88 days), and $29 \%$ re-initiating raloxifene (mean time from discontinuation to re-initiation: 109 days).

Predictors of non-persistence with the index medication were assessed in all study patients and in patients with 36 months of follow-up after initiating the index treatment (Table 4). Compared with oral bisphosphonates, the risk of non-persistence was 52 to $59 \%$ lower for denosumab, 17 to $32 \%$ lower for IV bisphosphonates, and 12 to $16 \%$ lower for raloxifene (all $p<0.0001$ ). The risk of non-persistence increased by $3 \%$ with each 5 -year increase in age after the age of 69 years $(p<0.0001)$. Black and Hispanic women were more likely to be non-persistent than White women ( $p<0.0001)$. Among women with 36 months of follow-up, Asian race was associated with a $4 \%$ reduction in risk of nonpersistence $(p=0.0025)$. A history of hip fracture at baseline was associated with a 10 to $12 \%$ lower risk of non-persistence (versus no historical hip fracture, $p<0.0001$ ). Similarly, a history of non-hip, non-vertebral fracture at baseline was associated with a $3 \%$ to $5 \%$ lower risk of non-persistence (versus no prior non-hip, non-vertebral fracture, $p 0.003$ and $p 0.02$, 
a

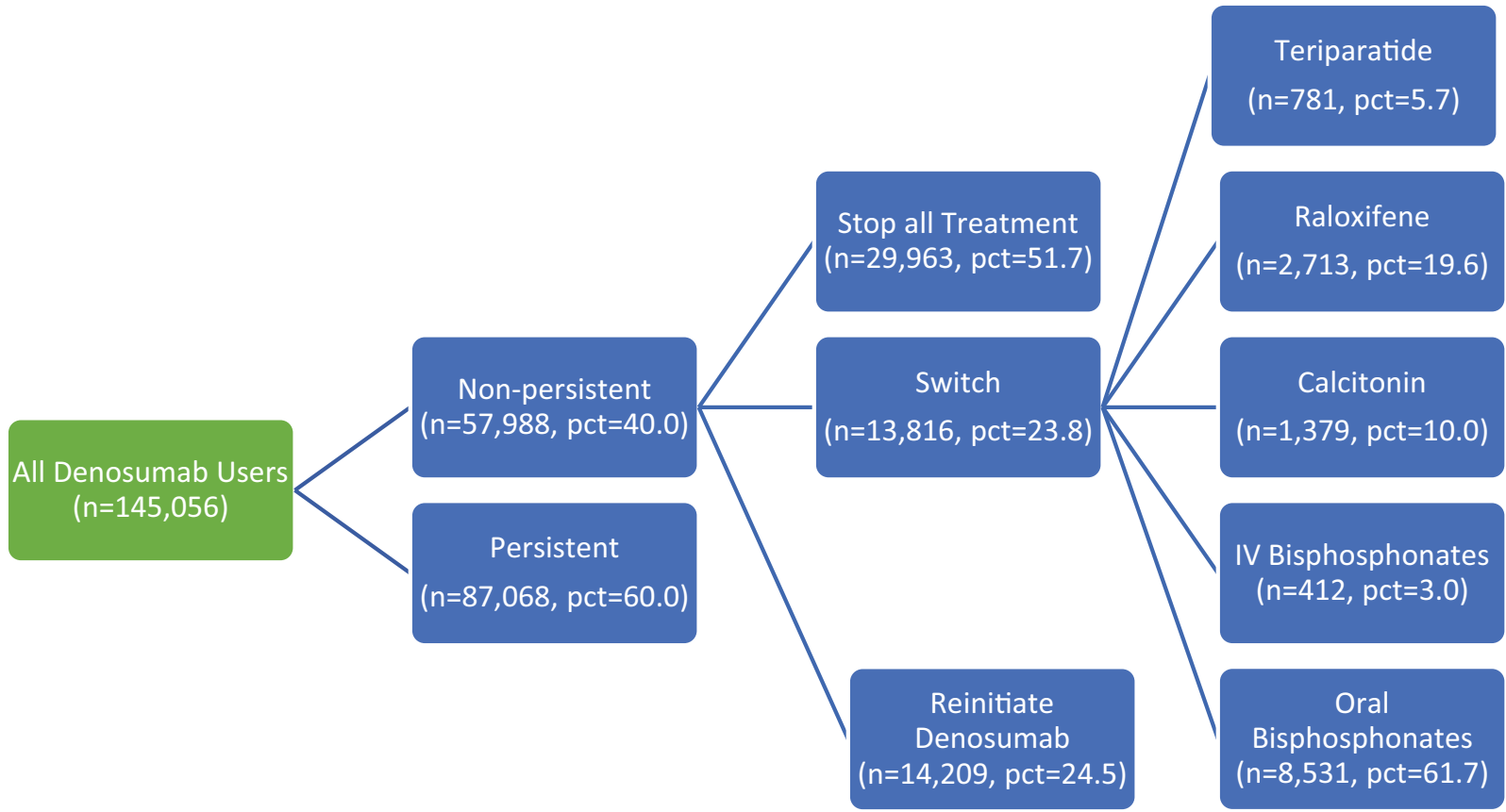

b

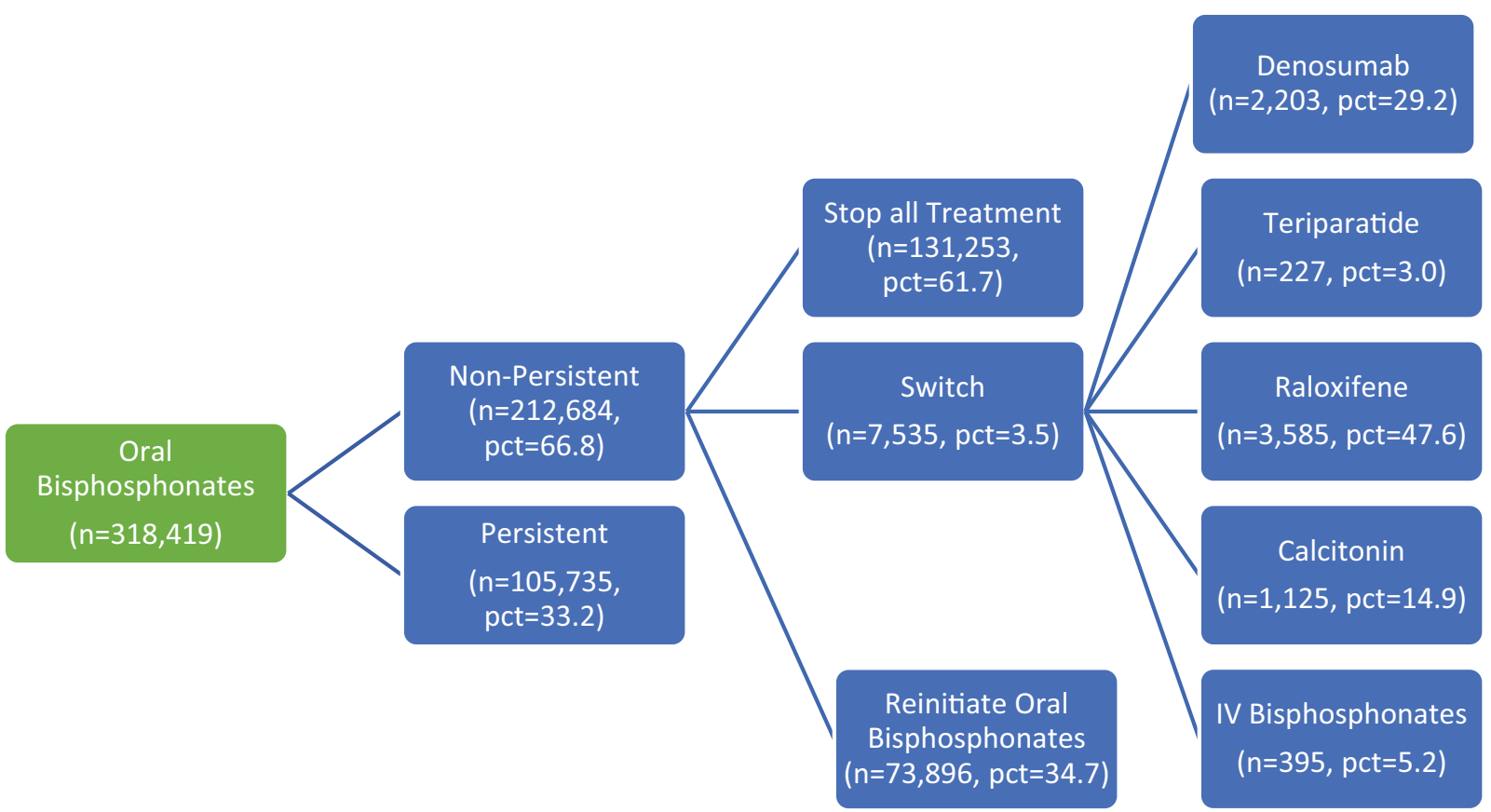

Fig. 2 Disposition through first treatment non-persistence. a. Denosumab users; b. Oral bisphosphonate users; c. IV bisphosphonate users; d. Raloxifene users 
C

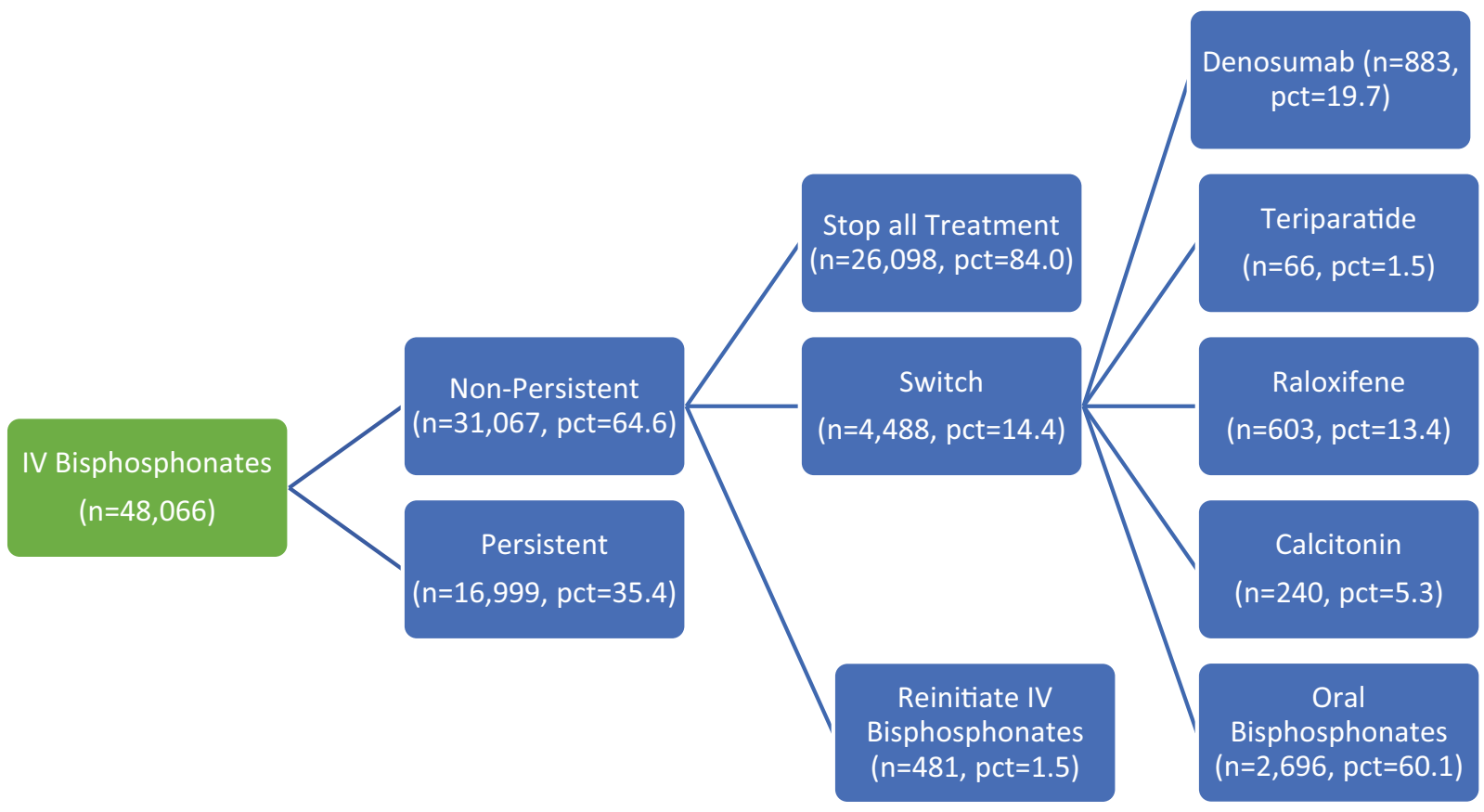

d

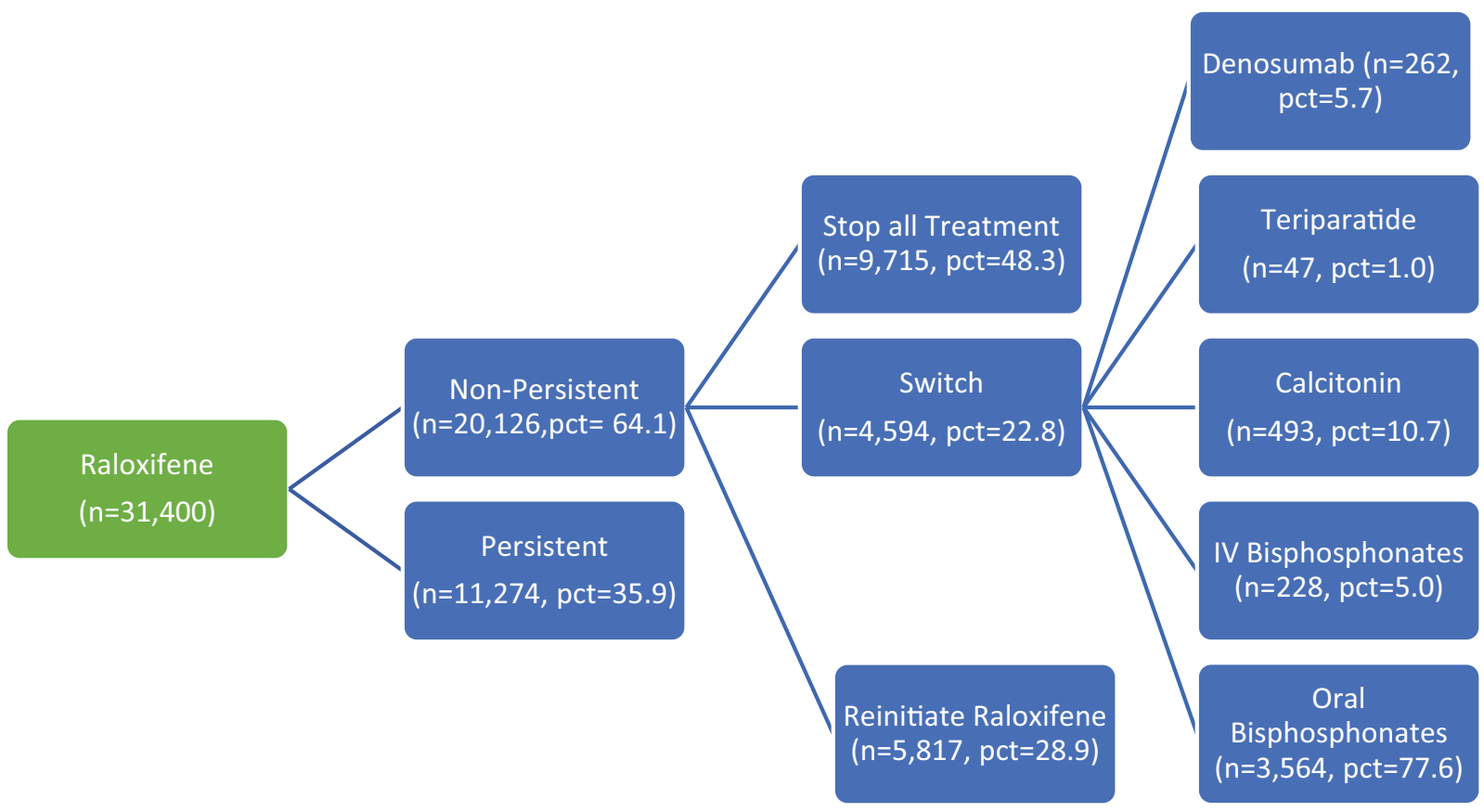

Fig. 2 (continued). 
Table 4 Predictors of non-persistence*

\begin{tabular}{|c|c|c|c|c|}
\hline \multirow[b]{2}{*}{ Baseline characteristic } & \multicolumn{2}{|l|}{ All patients } & \multicolumn{2}{|c|}{ Patients with 3 years follow-up } \\
\hline & HR $(95 \%$ CI) & $p$ Value & $\operatorname{HR}(95 \% \mathrm{CI})$ & $p$ Value \\
\hline \multicolumn{5}{|l|}{ Medication class } \\
\hline Oral & 1.00 (ref) & & 1.00 (ref) & \\
\hline Denosumab & $0.41(0.40,0.41)$ & $<.0001$ & $0.48(0.47,0.48)$ & $<.0001$ \\
\hline Raloxifene & $0.88(0.87,0.90)$ & $<.0001$ & $0.84(0.82,0.86)$ & $<.0001$ \\
\hline IV bisphosphonates & $0.68(0.67,0.69)$ & $<.0001$ & $0.83(0.81,0.84)$ & $<.0001$ \\
\hline \multicolumn{5}{|l|}{ Age group } \\
\hline $66-69$ & 1.00 (ref) & & 1.00 (ref) & \\
\hline $70-74$ & $1.04(1.03,1.05)$ & $<.0001$ & $1.03(1.01,1.05)$ & 0.0013 \\
\hline $75-79$ & $1.07(1.06,1.08)$ & $<.0001$ & $1.05(1.03,1.06)$ & $<.0001$ \\
\hline $80-84$ & $1.10(1.09,1.11)$ & $<.0001$ & $1.07(1.05,1.09)$ & $<.0001$ \\
\hline $85+$ & $1.13(1.12,1.14)$ & $<.0001$ & $1.12(1.09,1.14)$ & $<.0001$ \\
\hline \multicolumn{5}{|l|}{ Race } \\
\hline White & 1.00 (ref) & & 1.00 (ref) & \\
\hline Black & $1.12(1.10,1.13)$ & $<.0001$ & $1.11(1.08,1.14)$ & $<.0001$ \\
\hline Asian & $1.01(1.00,1.03)$ & 0.0767 & $0.96(0.94,0.99)$ & 0.0025 \\
\hline Hispanic & $1.18(1.16,1.20)$ & $<.0001$ & $1.19(1.16,1.23)$ & $<.0001$ \\
\hline Other & $1.02(1.00,1.04)$ & 0.0811 & $1.04(1.00,1.08)$ & 0.0251 \\
\hline Medicare-Medicaid dual eligible & $1.03(1.02,1.04)$ & $<.0001$ & $1.02(1.00,1.03)$ & 0.041 \\
\hline \multicolumn{5}{|l|}{ Inpatient hospital days } \\
\hline 0 & 1.00 (ref) & & 1.00 (ref) & \\
\hline $1-4$ & $0.99(0.98,1.01)$ & 0.3163 & $0.99(0.98,1.01)$ & 0.5817 \\
\hline $5+$ & $1.02(1.00,1.04)$ & 0.0398 & $1.00(0.97,1.03)$ & 0.9925 \\
\hline Transient ischemic attack & $0.98(0.97,0.99)$ & 0.0005 & $0.99(0.97,1.01)$ & 0.4852 \\
\hline Chronic heart failure & $1.06(1.04,1.08)$ & $<.0001$ & $1.05(1.02,1.09)$ & 0.0013 \\
\hline CVD & $1.03(1.02,1.04)$ & $<.0001$ & $1.04(1.02,1.05)$ & $<.0001$ \\
\hline COPD & $1.09(1.08,1.11)$ & $<.0001$ & $1.09(1.06,1.11)$ & $<.0001$ \\
\hline Anemia & $1.01(1.00,1.02)$ & 0.1111 & $1.01(0.99,1.02)$ & 0.5189 \\
\hline Depression & $1.02(1.01,1.03)$ & 0.0027 & $1.02(1.00,1.04)$ & 0.0547 \\
\hline Falls & $1.03(1.02,1.04)$ & $<.0001$ & $1.04(1.02,1.06)$ & $<.0001$ \\
\hline \multicolumn{5}{|l|}{ Medication classes group } \\
\hline$<5$ & 1.00 (ref) & & 1.00 (ref) & \\
\hline $5-10$ & $1.02(1.02,1.03)$ & $<.0001$ & $1.00(0.99,1.01)$ & 0.8246 \\
\hline $11+$ & $1.03(1.02,1.05)$ & $<.0001$ & $1.00(0.98,1.02)$ & 0.7908 \\
\hline NHNV fracture & $0.97(0.94,0.99)$ & 0.0029 & $0.95(0.91,0.99)$ & 0.0217 \\
\hline Hip fracture & $0.90(0.88,0.92)$ & $<.0001$ & $0.88(0.85,0.92)$ & $<.0001$ \\
\hline Vertebral fracture & $1.08(1.06,1.10)$ & $<.0001$ & $1.07(1.04,1.10)$ & $<.0001$ \\
\hline
\end{tabular}

*CMS region and Charlson Comorbidity Index were also included in the models as covariates, but are not shown for space considerations

respectively). By contrast, prior vertebral fracture was associated with a 7 to $8 \%$ increase in the risk of non-persistence (versus no historical vertebral fracture, $p<0.0001)$.

\section{Discussion}

The $100 \%$ Medicare osteoporosis sample includes data for a large, diverse population of postmenopausal women residing across the USA and is a unique data source from which to assess long-term persistence with osteoporosis medications in the real-world setting. In women with at least 36 months of follow-up, $73 \%$ of those who initiated denosumab for osteoporosis treatment were persistent with denosumab at 1 year, $50 \%$ at 2 years, and $38 \%$ at 3 years. The only other study, to our knowledge, that has looked at denosumab persistence over a 3-year period reported fairly similar persistence at 1 year $(77 \%)$ and higher persistence at 2 years and 3 years $(60 \%$ 
and $51 \%$, respectively) [29]. This difference may be partially explained by the fact that this earlier study was conducted within a single specialty community private practice where each patient had a 6-month follow-up appointment scheduled proactively at the time of each denosumab administration. In addition, the importance of treatment adherence (i.e., taking medication at the prescribed dose and dosing interval [13]) was discussed at each denosumab-related office visit, and patients received a reminder call just prior to each scheduled administration visit. Unfortunately, in the current study, data were not available to determine whether or not similar patient support activities were provided.

In our study, index medication persistence among women who initiated oral bisphosphonates (39\% at 1 year, $25 \%$ at 2 years, $17 \%$ at 3 years) was substantially lower than that observed for women who initiated on denosumab. This finding is consistent with a large body of literature documenting suboptimal persistence with oral bisphosphonates among postmenopausal women, a utilization pattern which leaves patients at elevated risk of fracture $[14,16,17,30]$. Medication characteristics and patients' understanding of the consequences of osteoporosis along with their perceptions of the risk/benefit trade-offs of pharmacologic osteoporosis therapies shape realworld persistence $[13,17,18]$. In addition, some physicians believe osteoporosis to be a "benign" or "inevitable" condition and/or that fragility fractures are rare with only a limited effect on patients [19-21]. Such attitudes likely contribute to undertreatment of osteoporosis and to patients' failure to initiate or persist with treatment, although the current study does not provide any information on how often primary nonadherence occurs.

Importantly, our examination of treatment patterns demonstrated that with the exception of raloxifene, the majority of patients who discontinued their index medications failed to either re-initiate the index agent or switch to another type of osteoporosis medication during their remaining follow-up. Among the denosumab patients who became non-persistent, $52 \%$ remained off treatment through the end of follow-up. The corresponding proportions for patients who were nonpersistent with their index oral bisphosphonates and index IV bisphosphonates and failed to resume osteoporosis treatment before the end of follow-up were $62 \%$ and $84 \%$, respectively.

Associations between better persistence and greater treatment benefit have been documented in the usual care setting, with claims-based studies showing persistent patients achieving up to a $29 \%$ greater fracture risk reduction than their less persistent counterparts $[14,15]$. It is, therefore, likely that the treatment effect was substantially blunted in the vast majority ( 83 to $94 \%$ ) of the patients in our study who initiated bisphosphonates, although this study was not designed to quantify the impact of persistence on fracture outcomes.
Results from the current study demonstrate the likely importance of persistence early in the patient's journey, since a substantial proportion of patients who were persistent in the first year or two on treatment were persistent in the second and third years, respectively. This trend was evident for all treatment classes except IV bisphosphonates and was strongest for denosumab and raloxifene. Although reasons for early discontinuation were unknown, touchpoints between healthcare providers and their postmenopausal patients who have recently initiated osteoporosis treatment may help uncover barriers to their patients' ability to comply with dosing instructions and frequency [31]. Such patient-provider interactions may be especially important in the early months, while the patient is adapting to a new medication regimen, and when side effects and issues with dosing instruction compliance are most likely to arise and potentially dissuade the patient from continuing therapy.

Managing non-persistence is especially important in patients initially treated with non-bisphosphonate osteoporosis medications such as denosumab and raloxifene as discontinuation without follow-on therapy results in rapid decreases in BMD, increased bone-turnover marker activity, and may ultimately increase fracture risk [32-36]. Timely re-initiation or switching to an alternative anti-resorptive therapy may reduce the risks of these potential adverse effects of discontinuing reversible drugs $[28,31]$.

Certain limitations should be considered in interpreting the results of this study. The claims data used in this study documents oral medication fills, but these data do not provide any information on whether or not the patient actually took the medications that were dispensed. It is likely, however, that patients who refilled the medication took the medication on hand prior to and thus necessitating a refill. Evaluation of persistence is complicated for zoledronic acid given its longer dosing interval, which dictates even longer-term follow-up studies to adequately assess persistence. These labeled dosing intervals reflect the long duration of anti-resorptive action for this agent, including evidence that bone-turnover markers may be suppressed by half for up to 5 years after a single infusion and trial data suggesting that a single baseline infusion and annual administrations over a 2 -year period may have similar effects on bone mineral density and boneturnover markers [32-35]. Fracture risk reduction has also been shown to persist for more than 1 year after a single 5$\mathrm{mg}$ dose of zoledronic acid in osteoporotic men and women [34], and in women with osteopenia, dosing every 18 months has been associated with reduced fracture risk during a 6-year interval [35]. The alternate persistence definition used in our sensitivity analysis was designed in consideration of this characteristic of zoledronic acid. It is reassuring that the patterns of persistence observed for denosumab and the comparator drugs with this alternate definition were consistent with results obtained with the primary persistence definition. It is also 
important to note that the potential for adverse effects if denosumab discontinuation occurs in the absence of followon anti-resorptive therapy (e.g., risk of multiple vertebral fractures) was not understood during the study period, and information related to this issue may influence treatment patterns in the future.

The standard limitations of claims data such as incomplete, inaccurate, or missing data are likely present in the source data, but these issues would not be expected to occur differentially by osteoporosis treatment class. Confounding by indication, such as differential disease severity among patients using different osteoporosis medications, is also possible and could impact patterns of osteoporosis medication use. Treatment non-persistence may be over-estimated in this study as our primary persistence definition included treatment discontinuations without further therapy as well as treatment discontinuations that occurred in conjunction with a switch to a different class of medication. This definition, however, is presented transparently, and in order to provide a more complete view of the patient journey, we have also provided data on patients' treatment disposition following initial treatment non-persistence. Reasons for treatment interruption could not be assessed within the current study scope, but this is an important topic for future research. The current study was designed to assess real-world persistence, but the clinical impact of non-persistence on fracture risk or biomarker change was not assessed. In addition, gender differences in medication persistence were not assessed since this study focused exclusively on postmenopausal women who initiated osteoporosis treatment. Finally, data analyzed covered 2010 to 2015 , and it is possible that treatment patterns as well as persistence may have changed over the years. Future research could provide insights using more recent data and potentially be designed to assess the clinical impact of non-persistence on fracture risk and biomarker change.

Study strengths include a rigorous assessment of long-term persistence across a broad range of common osteoporosis therapies in a large and diverse population of postmenopausal women. Results based on this broad population should generalize well to the national population of similarly aged women. In addition, the 3-year follow-up period in this study provides unique information on long-term persistence with anti-resorptive osteoporosis medications; this type of information is critical for building an understanding of the likely treatment benefit from real-world use since osteoporosis is a chronic disease that requires ongoing therapy. These results document better long-term persistence among patients who initiated osteoporosis treatment with denosumab, compared with patients who initiated other commonly used osteoporosis medications, including oral bisphosphonates; however, the observed level of medication persistence overall, even after considering treatment re-initiations and switches, indicates that there is a critical need for improvement. Further research is needed to better understand barriers to long-term persistence. Efforts to enhance physicians' and patients' understanding of the significance of osteoporosis and the role of persistence with pharmacologic therapy in fracture risk reduction will likely provide an important avenue for improving outcomes for patients with osteoporosis.

Supplementary Information The online version contains supplementary material available at https://doi.org/10.1007/s00198-021-05951-1.

Availability of data and material The Medicare data used for this study was licensed through the Centers for Medicare \& Medicaid Services and cannot be shared outside of that agreement.

Code availability Proprietary programming code was developed to analyze the Medicare data and not available outside of the study team.

Funding This study was supported by Amgen Inc.; Thousand Oaks, CA. Sally Wade of Wade Outcomes Research and Consulting provided writing support.

\section{Declarations}

Ethics approval Institutional Review Board approval was obtained through the Hennepin Healthcare Research Institute, Office for Human Subjects Research.

Conflicts of interest AS has received research/grant support from Radius and UCB, paid to MedStar; income from Agnovos, Amgen, Radius, and UCB for consulting/advisory boards; speaker's bureau fees from Amgen and Radius; and has also served on the National Osteoporosis Foundation Board of Trustees (non-remunerative). AY, RS, and SRG are Amgen employees and stockholders; JL and HY are employees of CDRG, and CDRG received research support from Amgen.

Open Access This article is licensed under a Creative Commons Attribution-NonCommercial 4.0 International License, which permits any non-commercial use, sharing, adaptation, distribution and reproduction in any medium or format, as long as you give appropriate credit to the original author(s) and the source, provide a link to the Creative Commons licence, and indicate if changes were made. The images or other third party material in this article are included in the article's Creative Commons licence, unless indicated otherwise in a credit line to the material. If material is not included in the article's Creative Commons licence and your intended use is not permitted by statutory regulation or exceeds the permitted use, you will need to obtain permission directly from the copyright holder. To view a copy of this licence, visit http:// creativecommons.org/licenses/by-nc/4.0/.

\section{References}

1. Cosman F, de Beur SJ, LeBoff MS, Lewiecki EM, Tanner B, Randall S, Lindsay R, National Osteoporosis Foundation (2014) Clinician's guide to prevention and treatment of osteoporosis. Osteoporos Int 25:2359-2381

2. Lewiecki EM, Wright NC, Curtis JR, Siris E, Gagel RF, Saag KG, Singer AJ, Steven PM, Adler RA (2018) Hip fracture trends in the United States, 2002 to 2015. Osteoporos Int 29:717-722 
3. International Osteoporosis Foundation (2020) Osteoporosis facts and statistics. www.iofbonehealth.org/facts-statistics. Accessed 17 September 2020

4. Bliuc D, Nguyen ND, Milch VE, Nguyen TV, Eisman JA, Center JR (2009) Mortality risk associated with low-trauma osteoporotic fracture and subsequent fracture in men and women. JAMA 301: 513-521

5. Hernlund E, Svedbom A, Ivergard M, Compston J, Cooper C, Stenmark J, McCloskey EV, Jonsson B, Kanis JA (2013) Osteoporosis in the European Union: medical management, epidemiology and economic burden. A report prepared in collaboration with the International Osteoporosis Foundation (IOF) and the European Federation of Pharmaceutical Industry Associations (EFPIA). Arch Osteoporos 8:136

6. Miller PD (2016) Underdiagnosis and undertreatment of osteoporosis: the battle to be won. J Clin Endocrinol Metab 101:852-859

7. Silverman SL, Minshall ME, Shen W, Harper KD, Xie S, HealthRelated Quality of Life Subgroup of the Multiple Outcomes of Raloxifene Evaluation S (2001) The relationship of health-related quality of life to prevalent and incident vertebral fractures in postmenopausal women with osteoporosis: results from the multiple outcomes of raloxifene evaluation study. Arthritis Rheum 44: 2611-2619

8. Strom O, Borgstrom F, Kanis JA, Compston J, Cooper C, McCloskey EV, Jonsson B (2011) Osteoporosis: burden, health care provision and opportunities in the EU: a report prepared in collaboration with the International Osteoporosis Foundation (IOF) and the European Federation of Pharmaceutical Industry Associations (EFPIA). Arch Osteoporos 6:59-155

9. Burge R, Dawson-Hughes B, Solomon DH, Wong JB, King A, Tosteson A (2007) Incidence and economic burden of osteoporosis-related fractures in the United States, 2005-2025. J Bone Miner Res 22:465-475

10. Singer A, Exuzides A, Spangler L, O'Malley C, Colby C, Johnston K, Agodoa I, Baker J, Kagan R (2015) Burden of illness for osteoporotic fractures compared with other serious diseases among postmenopausal women in the United States. Mayo Clin Proc 90: $53-62$

11. Kanis JA, McCloskey EV, Johansson H, Cooper C, Rizzoli R, Reginster JY, Scientific Advisory Board of the European Society for C, Economic Aspects of O, Osteoarthritis, the Committee of Scientific Advisors of the International Osteoporosis F (2013) European guidance for the diagnosis and management of osteoporosis in postmenopausal women. Osteoporos Int 24:23-57

12. Pazianas M, Abrahamsen B (2016) Osteoporosis treatment: bisphosphonates reign to continue for a few more years, at least? Ann N Y Acad Sci 1376:5-13

13. Cramer JA, Roy A, Burrell A, Fairchild CJ, Fuldeore MJ, Ollendorf DA, Wong PK (2008) Medication compliance and persistence: terminology and definitions. Value Health 11:44-47

14. Gold DT, Martin BC, Frytak JR, Amonkar MM, Cosman F (2007) A claims database analysis of persistence with alendronate therapy and fracture risk in post-menopausal women with osteoporosis. Curr Med Res Opin 23:585-594

15. Siris ES, Harris ST, Rosen CJ, Barr CE, Arvesen JN, Abbott TA, Silverman S (2006) Adherence to bisphosphonate therapy and fracture rates in osteoporotic women: relationship to vertebral and nonvertebral fractures from 2 US claims databases. Mayo Clin Proc 81:1013-1022

16. Liu J, Guo H, Rai P, Pinto L, Barron R (2018) Medication persistence and risk of fracture among female Medicare beneficiaries diagnosed with osteoporosis. Osteoporos Int 29:2409-2417

17. Karlsson L, Lundkvist J, Psachoulia E, Intorcia M, Strom O (2015) Persistence with denosumab and persistence with oral bisphosphonates for the treatment of postmenopausal osteoporosis: a retrospective, observational study, and a meta-analysis. Osteoporos Int 26:2401-2411

18. Pasco JA, Sanders KM, Hoekstra FM, Henry MJ, Nicholson GC, Kotowicz MA (2005) The human cost of fracture. Osteoporos Int 16:2046-2052

19. Fujiwara S, Zhao X, Teoh C, Jaffe DH, Taguchi Y (2019) Disease burden of fractures among patients with osteoporosis in Japan: health-related quality of life, work productivity and activity impairment, healthcare resource utilization, and economic costs. J Bone Miner Metab 37:307-318

20. Hiligsmann M, Salas M, Hughes DA, Manias E, Gwadry-Sridhar FH, Linck P, Cowell W (2013) Interventions to improve osteoporosis medication adherence and persistence: a systematic review and literature appraisal by the ISPOR Medication Adherence \& Persistence Special Interest Group. Osteoporos Int 24:2907-2918

21. Caro JJ, Ishak KJ, Huybrechts KF, Raggio G, Naujoks C (2004) The impact of compliance with osteoporosis therapy on fracture rates in actual practice. Osteoporos Int 15:1003-1008

22. Cornelissen D, de Kunder S, Si L, Reginster JY, Evers S, Boonen A, Hiligsmann M, European Society for C, Economic Aspect of Osteoporosis O, Musculoskeletal D (2020) Interventions to improve adherence to anti-osteoporosis medications: an updated systematic review. Osteoporos Int 31:1645-1669

23. Salminen H, Piispanen P, Toth-Pal E (2019) Primary care physicians' views on osteoporosis management: a qualitative study. Arch Osteoporos 14:48

24. Alami S, Hervouet L, Poiraudeau S, Briot K, Roux C (2016) Barriers to effective postmenopausal osteoporosis treatment: a qualitative study of patients' and practitioners' views. PLoS One 11: e0158365

25. Lems WF, Raterman HG (2017) Critical issues and current challenges in osteoporosis and fracture prevention. An overview of unmet needs. Ther Adv Musculoskelet Dis 9:299-316

26. Fahrleitner-Pammer A, Papaioannou N, Gielen E, Feudjo Tepie M, Toffis C, Frieling I, Geusens P, Makras P, Boschitsch E, Callens J, Anastasilakis AD, Niedhart C, Resch H, Kalouche-Khalil L, Hadji P (2017) Factors associated with high 24-month persistence with denosumab: results of a real-world, non-interventional study of women with postmenopausal osteoporosis in Germany, Austria, Greece, and Belgium. Arch Osteoporos 12:58

27. Silverman SL, Siris E, Belazi D, Recknor C, Papaioannou A, Brown JP, Gold DT, Lewiecki EM, Quinn G, Balasubramanian A, Yue S, Stolshek B, Kendler DL (2018) Persistence at 24 months with denosumab among postmenopausal women with osteoporosis: results of a prospective cohort study. Arch Osteoporos 13:85

28. Silverman SL, Siris E, Kendler DL, Belazi D, Brown JP, Gold DT, Lewiecki EM, Papaioannou A, Simonelli C, Ferreira I, Balasubramanian A, Dakin P, Ho P, Siddhanti S, Stolshek B, Recknor C (2015) Persistence at 12 months with denosumab in postmenopausal women with osteoporosis: interim results from a prospective observational study. Osteoporos Int 26:361-372

29. Borek DM, Smith RC, Gruber CN, Gruber BL (2019) Long-term persistence in patients with osteoporosis receiving denosumab in routine practice: 36 -month non-interventional, observational study. Osteoporos Int 30:1455-1464

30. Gold DT, Silverman S (2006) Review of adherence to medications for the treatment of osteoporosis. Curr Osteoporos Rep 4:21-27

31. Lee S, Glendenning P, Inderjeeth CA (2011) Efficacy, side effects and route of administration are more important than frequency of dosing of anti-osteoporosis treatments in determining patient adherence: a critical review of published articles from 1970 to 2009 . Osteoporos Int 22:741-753

32. Tsourdi E, Langdahl B, Cohen-Solal M, Aubry-Rozier B, Eriksen EF, Guanabens N, Obermayer-Pietsch B, Ralston SH, Eastell R, Zillikens MC (2017) Discontinuation of denosumab therapy for 
osteoporosis: a systematic review and position statement by ECTS. Bone 105:11-17

33. Anastasilakis AD, Evangelatos G, Makras P, Iliopoulos A (2020) Rebound-associated vertebral fractures may occur in sequential time points following denosumab discontinuation: need for prompt treatment re-initiation. Bone Rep 12:100267

34. Tripto-Shkolnik L, Fund N, Rouach V, Chodick G, Shalev V, Goldshtein I (2020) Fracture incidence after denosumab discontinuation: real-world data from a large healthcare provider. Bone 130: 115150

35. Dennison EM, Cooper C, Kanis JA, Bruyere O, Silverman S, McCloskey E, Abrahamsen B, Prieto-Alhambra D, Ferrari S,
Group IOFEQoLW (2019) Fracture risk following intermission of osteoporosis therapy. Osteoporos Int 30:1733-1743

36. Siris ES, Harris ST, Eastell R, Zanchetta JR, Goemaere S, DiezPerez A, Stock JL, Song J, Qu Y, Kulkarni PM, Siddhanti SR, Wong M, Cummings SR, Continuing Outcomes Relevant to Evista (CORE) Investigators (2005) Skeletal effects of raloxifene after 8 years: results from the continuing outcomes relevant to Evista (CORE) study. J Bone Miner Res 20:1514-1524

Publisher's note Springer Nature remains neutral with regard to jurisdictional claims in published maps and institutional affiliations. 